\title{
Prescription for probabilities in eternal inflation
}

\author{
Jaume Garriga \\ Institute of Cosmology, Department of Physics and Astronomy, Tufts University, Medford, Massachusetts 02155 \\ and IFAE, Departament de Física, Universitat Autònoma de Barcelona, 08193 Bellaterra, Barcelona, Spain
}

Alexander Vilenkin

Institute of Cosmology, Department of Physics and Astronomy, Tufts University, Medford, Massachusetts 02155

(Received 23 March 2001; published 12 June 2001)

\begin{abstract}
Some of the parameters we call "constants of nature" may in fact be variables related to the local values of some dynamical fields. During inflation, these variables are randomized by quantum fluctuations. In cases when the variable in question (call it $\chi$ ) takes values in a continuous range, all thermalized regions in the universe are statistically equivalent, and a gauge invariant procedure for calculating the probability distribution for $\chi$ is known. This is the so-called "spherical cutoff method." In order to find the probability distribution for $\chi$ it suffices to consider a large spherical patch in a single thermalized region. Here, we generalize this method to the case when the range of $\chi$ is discontinuous and there are several different types of thermalized region. We first formulate a set of requirements that any such generalization should satisfy, and then introduce a prescription that meets all the requirements. We finally apply this prescription to calculate the relative probability for different bubble universes in the open inflation scenario.
\end{abstract}

DOI: $10.1103 /$ PhysRevD.64.023507

PACS number(s): $98.80 . \mathrm{Cq}$

\section{INTRODUCTION}

The parameters we call "constants of nature" may in fact be variables related to the local values of certain dynamical fields. For example, what we perceive as a cosmological constant could be a potential $U(\chi)$ of some slowly varying field $\chi(x)$. If this potential is very flat, so that the evolution of $\chi$ is much slower than the Hubble expansion, then observations will not distinguish between $U(\chi)$ and a true cosmological constant. Observers in different parts of the universe could then measure different values of $U(\chi)$.

Spatial variation of the fields $\chi_{a}$ associated with the "constants" can naturally arise in the framework of inflationary cosmology [1]. The dynamics of light scalar fields during inflation are strongly influenced by quantum fluctuations, so different regions of the universe thermalize with different values of $\chi_{a}$. An important question is whether or not we can predict the values of the "constants" we are most likely to observe. In more general terms, we are interested in determining the probability distribution $\mathcal{P}(\chi)$ for us to measure certain values of $\chi_{a}$. The answer to this question must involve anthropic considerations to some extent. The laws of physics may be sufficient to determine the range and even the spacetime distribution of the variables $\chi_{a}$. However, some values of $\chi_{a}$ that are physically allowed may be incompatible with the very existence of observers, and in this case they will never be measured. The relevant question is then how to assign a weight to this selection effect.

The inflationary scenario implies a very large universe inhabited by numerous civilizations that will measure different values of $\chi_{a}$. We can define the probability $\mathcal{P}(\chi) d \chi_{1} \ldots d \chi_{k}$ for $\chi_{a}$ to be in the intervals $d \chi_{a}$ as being proportional to the number of civilizations which will measure $\chi_{a}$ in that interval [2]. This includes all present, past and future civilizations; in other words, it is the number of civilizations throughout the entire spacetime, rather than at a particular moment of time. Assuming that we are a typical civilization, we can expect to observe $\chi_{a}$ near the maximum of $\mathcal{P}(\chi)$ [3]. The assumption of being typical has been called the "principle of mediocrity" in Ref. [2].

An immediate objection to this approach is that we are ignorant about the origin of life, let alone intelligence, and therefore the number of civilizations cannot be calculated. But even if this were true, the approach could still be used to find the probability distribution for parameters that do not affect the physical processes involved in the evolution of life. The cosmological constant $\Lambda$, the density parameter $\Omega$ and the amplitude of density fluctuations $Q$ are examples of such parameters. Assuming that our fields $\chi_{a}$ belong to this category, the probability for a civilization to evolve on a suitable planet is then independent of $\chi_{a}$, and instead of the number of civilizations we can use the number of habitable planets or, as a rough approximation, the number of galaxies. Thus, we can write

$$
\mathcal{P}(\chi) d^{k} \chi \propto d \mathcal{N}
$$

where $d \mathcal{N}$ is the number of galaxies that are going to be formed in regions where $\chi_{a}$ take values in the intervals $d \chi_{a}$.

The probability distribution (1) based on plain galaxy counting is interesting in its own right, since it gives a quantitative characterization of the large scale properties of the universe. Thus, the general rules for calculating (1) are worth investigating quite independently from anthropic considerations. These considerations can always be included a posteriori, as an additional factor giving the number of civilizations per galaxy.

The number of galaxies $d \mathcal{N}(\chi)$ in Eq. (1) is proportional to the volume of the comoving regions where $\chi_{a}$ take specified values and to the density of galaxies in those regions. The volumes and the densities can be evaluated at any time, as long as we include both galaxies that were formed in the 


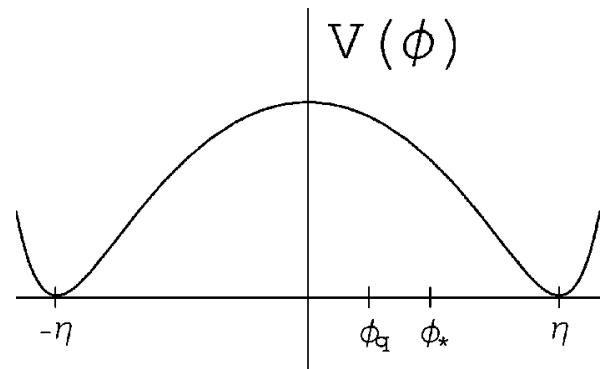

FIG. 1. Symmetric double well inflaton potential.

past and those that are going to be formed in the future. It is convenient to evaluate the volumes and the densities at the time when inflation ends and vacuum energy thermalizes, that is, on the thermalization surface $\Sigma_{*}$. Then we can write

$$
\mathcal{P}(\chi) \propto \nu(\chi) \mathcal{P}_{*}(\chi) .
$$

Here, $\mathcal{P}_{*}(\chi) d^{k} \chi$ is proportional to the volume of thermalized regions where $\chi_{a}$ take values in the intervals $d \chi_{a}$, and $\nu(\chi)$ is the number of galaxies that form per unit thermalized volume with cosmological parameters specified by the values of $\chi_{a}$. The calculation of $\nu(\chi)$ is a standard astrophysical problem which is completely unrelated to the calculation of the volume factor $\mathcal{P}_{*}(\chi)$, and which does not pose difficulties of principle.

The meaning of Eq. (1) is unambiguous in models where the total number of galaxies in the universe is finite. Otherwise, one has to introduce some cutoff and define the ratio of probabilities for the intervals $d^{n} \chi^{(1)}$ and $d^{n} \chi^{(2)}$ as the ratio of the galaxy numbers $d \mathcal{N}^{(1)} / d \mathcal{N}^{(2)}$ in the limit when the cutoff is removed. However, this limiting procedure has proved to be rather non-trivial, and a general method that would apply to all possible eternally inflating scenarios has not yet been found.

The situation is relatively straightforward in the case of an infinite universe which is more or less homogeneous on very large scales. One can evaluate the ratio $d \mathcal{N}^{(1)} / d \mathcal{N}^{(2)}$ in a large comoving volume $\mathcal{V}$ and then take the limit as $\mathcal{V} \rightarrow \infty$. The result is expected to be independent of the limiting procedure; for example, it should not depend on the shape of the volume $\mathcal{V}$. (It is assumed that the volume selection is unbiased, that is, that the volume $\mathcal{V}$ is not carved to favor some values of $\chi_{a}$ at the expense of other values.)

However, the situation with an infinite universe which is homogeneous on very large scales is not generic in the context of inflation. Most inflationary scenarios predict that inflation is eternal to the future, and therefore the universe is never completely thermalized $[7,8]$ (for a recent review of eternal inflation see [9]). An example that is particularly relevant to the subject of the present paper is given by the double well inflaton potential depicted in Fig. 1. The inflaton $\phi$ can thermalize in two different vacua, labeled by $\eta_{1}$ and $\eta_{2}$. The spacetime distribution of the field in this model is depicted in Fig. 2. There are thermalized regions of two types, characterized by the inflaton vacuum expectation value $\eta_{i}$. Thermalized regions with $\phi=\eta_{1}$ are disconnected from thermalized regions with $\phi=\eta_{2}$. Both types of region

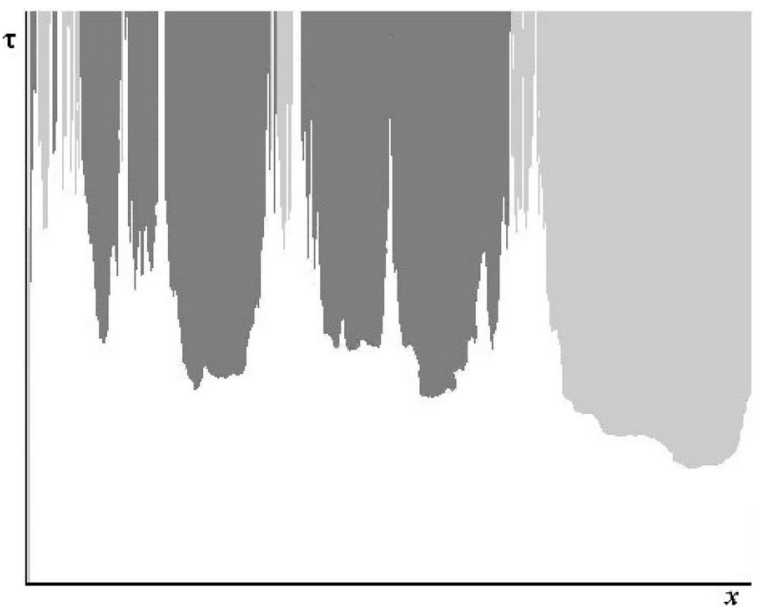

FIG. 2. A numerical simulation of the spacetime structure of an inflating universe [11]. The simulation corresponds to a double-well inflaton potential, with two degenerate minima where the inflaton takes the values $\pm \eta$. Inflating regions are white, while thermalized regions with inflaton values equal to $+\eta$ and $-\eta$ are shown with different shades of grey.

are separated by inflating domain walls $[13,14]$, and so the universe is never completely thermalized. Each thermalization surface is infinite, so it will contain an infinite number of galaxies. Moreover, there are an infinite number of thermalized regions of each type. Therefore, the implementation of Eq. (1) for calculating probabilities requires the comparison of infinite sets of galaxies which lie in disconnected regions of the universe. A similar spacetime structure is obtained if we make the inflaton potential periodic by identifying the two minima. In this case there are still two different types of thermalization surface, characterized by the two topologically different paths that one can take from the top of the potential to the thermalized region. Although the particle physics parameters of both types of thermalized region are guaranteed to be the same, other cosmological parameters such as the spectrum of density perturbations or the spatial distribution of an effective cosmological constant will in general be different.

In any model of eternal inflation, the volumes of both inflating and thermalized regions grow exponentially with time and the number of galaxies grows without bound, even in a region of a finite comoving size. One can try to deal with this problem by introducing a time cutoff and including only regions that thermalized prior to some moment of time $t_{c}$, with the limit $t_{c} \rightarrow \infty$ at the end. One finds, however, that the resulting probability distributions are extremely sensitive to the choice of the time coordinate $t$ [4]. Coordinates in general relativity are arbitrary labels, and such gauge dependence of the results casts doubt on any conclusion reached using this approach.

A resolution of the gauge dependence problem was proposed in Ref. [10] and subsequently developed in $[11,12]$. The proposed method can be summarized as follows. Let us first assume that inflating and thermalized regions of spacetime are separated by a single thermalization surface $\Sigma_{*}$. The problem with the constant-time cutoff procedures is that they cut the surface $\Sigma_{*}$ in a biased way, favoring certain 
values of $\chi$ and disfavoring other values. We thus need a portion of $\Sigma_{*}$ selected without bias. The simplest strategy is to use a "spherical" cutoff. Choose an arbitrary point $P$ on $\Sigma_{*}$. Define a sphere of radius $R$ to include all points $Q$ whose distance from $P$ along $\Sigma_{*}$ is $d(Q, P) \leqslant R$. We can use Eq. (1) to evaluate the probability distribution $\mathcal{P}(\chi)$ in a spherical volume of radius $R_{c}$ and then let $R_{c} \rightarrow \infty$. If the fields $\chi_{a}$ vary in a finite range, they will run through all of their values many times in a spherical volume of sufficiently large radius. We expect, therefore, that the distribution $\mathcal{P}(\chi)$ will rapidly converge as the cutoff radius $R_{c}$ is increased. We expect also that the resulting distribution will be independent of the choice of point $P$ that serves as the center of the sphere. The same procedure can be used for fields with an infinite range of variation, provided that the probability distributions for $\chi_{a}$ are concentrated within a finite range, with a negligible probability of finding $\chi_{a}$ very far away from that range.

Suppose now that there is an infinite number of disconnected thermalization surfaces, as happens generically in eternal inflation. Further, we assume that the variables $\chi_{a}$ of interest are such that their whole range of values is allowed to occur in a single thermalized region (this is the case, for instance, for the slowly varying field $\chi$ which plays the role of a cosmological constant), and that, unlike the case of the double well potential in Fig. 1, there is only one type of thermalized region. We can then pick an arbitrary connected component of $\Sigma_{*}$ and apply the spherical cutoff prescription described above. Since the inflationary dynamics of the fields $\chi_{a}$ have a stochastic nature, the distributions of $\chi_{a}$ on different connected components of $\Sigma_{*}$ should be statistically equivalent, and the resulting probability distribution $\mathcal{P}(\chi)$ should be the same for all components. This has been verified both analytically and numerically in [11].

The main shortcoming of the spherical cutoff prescription is that as it stands it cannot be applied to models where the inflaton potential has a discrete set of minima, as in the example shown in Fig. 1. More precisely, the problem arises when the minima are separated by inflating domain walls $[13,14]$. In this case, we can introduce a discrete variable $n$ labeling different minima. Each connected component of the thermalization surface $\Sigma_{*}$ will be characterized by a single value of $n$ (unless the different minima can be separated by non-inflating domain walls) and it is clear that the probability distribution for $n$ cannot be determined by studying one such component.

The purpose of this paper is to propose a generalization of the spherical cutoff prescription that would be applicable in the general case. We begin in Sec. II by formulating the requirements that we believe any such proposal should satisfy. We require that it should be gauge-independent and should reduce to the spherical cutoff prescription in the absence of discrete variables. Moreover, we consider a class of asymmetric double-well potentials for which the probabilities can be calculated in a well-motivated way. We then require that the general prescription should give the same result for this class of potentials. In Sec. III we propose a prescription that satisfies all of the above requirements. We use this prescription in Sec. IV to calculate probabilities for

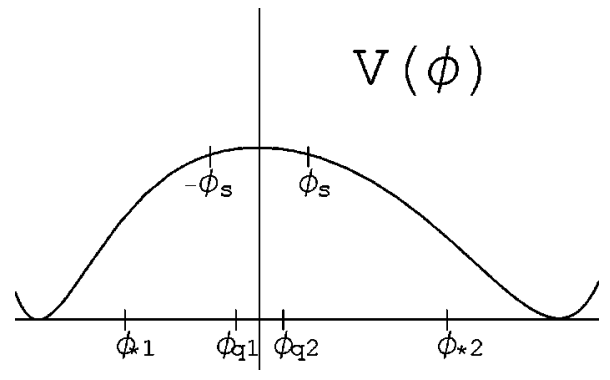

FIG. 3. Asymmetric double well potential which is symmetric near the maximum in the range $-\phi_{s}<\phi<\phi_{s}$.

bubble universes in the open inflation scenario. Our conclusions are summarized and discussed in Sec. V.

\section{REQUIREMENTS}

Suppose that the inflaton potential has $N$ minima labeled by $i=1,2, \ldots, N$, so that there are $N$ different types of thermalized region. Suppose also that there is a set of scalar fields $\chi_{a}$ which take a continuous range of values in all types of thermalized region. Our goal is to calculate the probability distribution

$$
\mathcal{P}_{i}(\chi)=P_{i} \hat{\mathcal{P}}_{i}(\chi)
$$

Here, $\hat{\mathcal{P}}_{i}(\chi)$ is the normalized distribution for $\chi$ within the $n$th type of thermalized region,

$$
\int d^{k} \chi \hat{\mathcal{P}}_{i}(\chi)=1
$$

and $P_{i}$ is the probability for an observer to find herself in a thermalized region of type $i$.

We begin with the obvious requirements that the probabilities (3) should be gauge-invariant and should satisfy the normalization condition

$$
\sum_{i} P_{i}=1
$$

Next, we require that for $N=1$ the prescription should reduce to the spherical cutoff prescription. This implies that the spherical cutoff should be used for the calculation of the individual distributions $\hat{\mathcal{P}}_{i}(\chi)$ within each type of thermalized region. What remains to be determined are the relative probabilities of different types of regions, $P_{i}$.

Finally, we introduce a class of inflaton potentials for which we believe there is a well-motivated answer for the probabilities. Consider first a symmetric double-well potential, $V(\phi)=V(-\phi)$, with a maximum at $\phi=0$ and two minima at $\phi_{1,2}= \pm \eta$, as in Fig. 1. Clearly, in this case the symmetry of the problem dictates that $P_{1}=P_{2}=0.5$.

Next, we consider an asymmetric double-well, which however is symmetric in some range of $\phi$ near the maximum, $|\phi|<\phi_{s}$ (see Fig. 3). Quantum fluctuations of $\phi$ dominate the dynamics in the range $|\phi| \lesssim \phi_{q}$, while for $\phi \gg \phi_{q}$ the evolution is essentially deterministic. We shall assume 
that $\phi_{s}$ is in the deterministic slow-roll regime, $\phi_{s} \gg \phi_{q}$. Let us consider constant- $\phi$ surfaces $\phi= \pm \phi_{s}$. These are infinite spacelike surfaces that have $|\phi|<\phi_{s}$ everywhere in their past. Since the potential $V(\phi)$ is symmetric in this range of $\phi$, all these surfaces are statistically equivalent. The symmetry of the problem suggests that the probabilities $P_{1,2}$ can be calculated by sampling comoving spheres of equal radius on the two types of surface. The ratio of the probabilities will then be

$$
P_{1} / P_{2}=N_{1} / N_{2},
$$

where $N_{1}, N_{2}$ are average numbers of galaxies that will form in large comoving spherical regions which have equal radii at $\phi= \pm \phi_{s}$. If we assume for simplicity that the two types of regions have identical physics at thermalization and afterwards, then the difference between $N_{1}$ and $N_{2}$ can only be due to the different inflationary expansion factors $Z_{i}$ characterizing the evolution from $\pm \phi_{s}$ to the thermalization points $\phi_{* i}$. We then have $N_{i} \propto Z_{i}^{3}$ and

$$
P_{1} / P_{2}=\left(Z_{1} / Z_{2}\right)^{3} \text {. }
$$

We shall require that the general prescription for probabilities should reproduce Eq. (7) in the case of asymmetric double-well potentials of the type we discussed here.

\section{THE PROPOSAL}

In the general case, the inflaton potential has no symmetries to guide our selection of the equal- $\phi$ surfaces on which to calculate probabilities. Suppose the potential has a maximum, which we choose to be at $\phi=0$, and two minima with thermalization points at $\phi_{* 1}$ and $\phi_{* 2}$. We can then choose some arbitrary values $\phi_{1}$ and $\phi_{2}$ in the slow roll ranges of $\phi$ adjacent to $\phi_{* 1}$ and $\phi_{* 2}$, respectively, and calculate the probabilities by sampling the surfaces $\phi=\phi_{i}$. Imagine for a moment that the number of thermalized regions of both types and the number of galaxies in each region are all finite. Then we could write

$$
\frac{P_{1}}{P_{2}}=\frac{p_{1}}{p_{2}} \frac{N_{1}}{N_{2}} .
$$

Here, $p_{i}$ is the probability for a randomly selected thermalized region to be of type $i$ and $N_{i}$ is the average number of galaxies in a type- $i$ region. In our case, however, the number of thermalized regions and the number of galaxies in each region are infinite, so the definitions of the probabilities $p_{i}$ and of the ratio $N_{1} / N_{2}$ are problematic.

It has been remarked $[6,9]$ that the problem of determining $p_{i}$ is similar to the problem of calculating the probability $p_{\text {even }}$ that a randomly selected integer is even. If we take a long stretch of the natural series

$$
1,2,3, \ldots
$$

it will have nearly equal quantities of even and odd numbers, suggesting that $p_{\text {even }}=1 / 2$. However, the series can be reordered as

$$
1,2,4,3,6,8,5, \ldots
$$

and the same calculation would give $p_{\text {even }}=2 / 3$. It is clear that by appropriately ordering the series one can obtain any answer for $p_{\text {even }}$ between 0 and 1 . This seems to suggest that the probabilities $p_{i}$ are hopelessly ill-defined.

We note, however, that the situation with the natural series is not as bad as it may seem. The series has a natural ordering in which the nearest neighbors of each number differ from that number by 1 , and we can require that our sampling procedure should respect this natural "topology." Then we have $p_{\text {even }}=1 / 2$, which is the answer that one intuitively expects. With an infinite number of thermalized regions of different types, one could also order the list of regions in a way that would give any desired result for $p_{1} / p_{2}$. But again one can hope that this ambiguity can be removed if we require that our sampling procedure should reflect the spatial distribution of the regions.

The ratio $N_{1} / N_{2}$ can be calculated by counting galaxies in spheres located in regions of the two types. In the doublewell example of Sec. II, there is complete symmetry between the surfaces $\phi=\phi_{s}$ and $\phi=-\phi_{s}$. We expect, therefore, that $p_{1}=p_{2}$ and we choose the radii of the spheres to be equal at $\phi= \pm \phi_{s}$. However, it is not clear what sets the relative size of the spheres in the absence of symmetry. We shall now describe the method we propose for evaluating $p_{i}$ and $N_{1} / N_{2}$ in the general case.

We start by noting that there is one thing that thermalized regions of the two types have in common. In their past they all went through a period of stochastic inflation, with the inflaton field $\phi$ undergoing a random walk near the top of the potential. Our idea is to use some markers from this early era for the calculation of probabilities.

Let us imagine that markers are point objects and that they are produced at a constant rate per unit spacetime volume in inflating regions where $\phi$ is at the top of its potential, $V(\phi) \approx V_{\max }$. After that, the markers evolve as comoving test particles and eventually end up in a thermalized region of one type or the other. We shall define $p_{i}$ as the fraction of markers that end up in regions of type $i$. Furthermore, the reference length scale on which the number of galaxies is counted in a region of type $i$ will be set by the average distace $d_{i}$ between markers in that type of region. In other words, the galaxies are counted in spheres of radii $R_{1}$ and $R_{2}$ such that $R_{1} / R_{2}=d_{1} / d_{2}$. To summarize, we propose that the relative probabilities for the constants of nature are given by Eq. (3) with

$$
\frac{P_{1}}{P_{2}}=\frac{p_{1} n_{2}}{p_{2} n_{1}} \frac{\nu_{1}}{\nu_{2}},
$$

where $n_{i} \propto d_{i}^{-3}$ is the mean number density of markers in regions of type $i$ and $\nu_{i}$, given by

$$
\nu_{i}^{-1}=\int \hat{\mathcal{P}}_{i}(\chi) \nu_{i}^{-1}(\chi) d \chi
$$




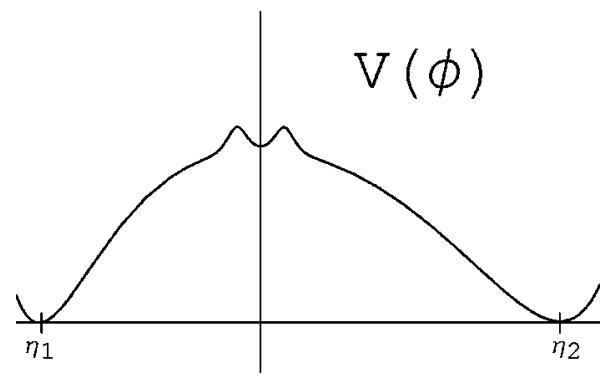

FIG. 4. Asymmetric inflaton potential with a metastable vacuum at the top, which replaces the quantum diffusion region of Fig. 3 .

is the mean density of galaxies in that type of region. As mentioned in the Introduction, the calculation of $\nu_{i}$ is a standard astrophysical problem which we shall not be concerned with in this paper.

A physical counterpart to the production of our idealized markers is quantum nucleation of black holes in an inflating universe. Black holes are produced at a rate [15] $r \propto \exp$ $[-1 / 8 V(\phi)]$ that grows exponentially with $V(\phi)$, so that by far the highest rate is achieved at $V(\phi)=V_{\max }$. Here, we prefer not to identify markers with black holes and to think of them as of idealized point objects. This frees us from concerns such as the contribution to black hole production from regions that are not at the top of the potential, black hole evaporation, etc. The reality and observability of markers is not really an issue, since the comparison of causally disconnected thermalized regions is certainly a gedanken experiment.

The average comoving distance between the markers in a thermalized region is manifestly gauge invariant, and so is the ratio $n_{2} / n_{1}$ in Eq. (11). The quantities $p_{i}$ are also gaugeindependent, and thus the probabilities $P_{i}$ in (11) should be gauge-invariant.

It is also easy to verify that the above proposal gives the expected result (7) for the probabilities when the inflaton potential is symmetric in the diffusion range of $\phi$. In this case, the average distance between markers on the surface $\phi=\phi_{s}$ is the same as that on $\phi=-\phi_{s}$ (with $\phi_{s}$ defined in Sec. II). Hence, the distances between markers at thermalization may differ only due to the difference in the expansion factors from $\pm \phi_{s}$ to $\phi_{* i}$,

$$
d_{1} / d_{2}=Z_{1} / Z_{2},
$$

and Eq. (7) follows immediately.

\section{OPEN INFLATION}

As an illustration of the method we shall now calculate probabilities for a model of "open inflation" [16-18]. We assume an inflaton potential of the form shown in Fig. 4. It has a metastable false vacuum at $\phi=0$ which is separated by potential barriers from two slow roll regions on the left and on the right. The false vacuum decays through bubble nucleation, and the inflaton rolls towards the true vacuum inside the bubbles. Comoving observers inside each bubble would, after thermalization of the inflaton, see themselves in an open homogeneous universe. (Hence the name "open infla-

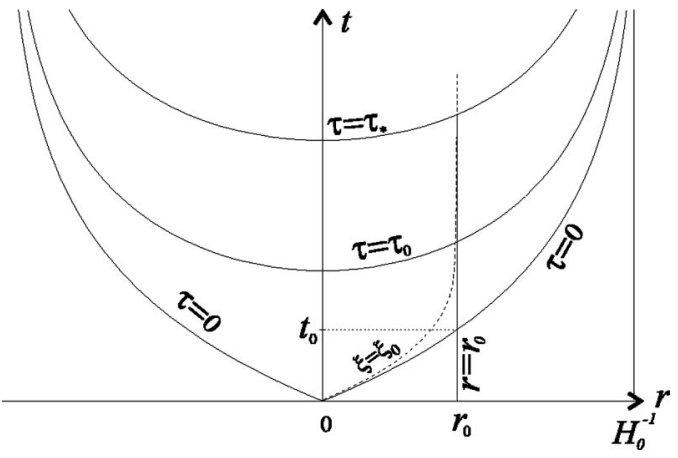

FIG. 5. Spacetime geometry of the bubble in comoving coordinates. Here, $r=|\mathbf{x}|$, and various $\tau=$ const surfaces of the open Robertson-Walker universe inside the bubble are shown, as well as one of the $\xi=$ const curves.

tion." ) If the bubble nucleation rate is not too high, bubble collisions are rare and inflation is eternal. Assuming that the two types of bubble have identical low-energy physics, we would like to find the probability for an observer to find herself in one type of bubble or the other.

In false vacuum regions outside bubbles the metric is de Sitter,

$$
d s^{2}=d t^{2}-e^{2 H_{0} t} d \mathbf{x}^{2}
$$

where $H_{0}=8 \pi G V_{0} / 3$ and $V_{0}=V(0)$ is the false vacuum energy density. The bubble interior has the geometry of an open Robertson-Walker universe,

$$
d s^{2}=d \tau^{2}-a^{2}(\tau)\left[d \xi^{2}+\sinh ^{2} \xi d \Omega^{2}\right] .
$$

After nucleation, the bubble wall expands rapidly approaching the speed of light. If the initial bubble size is much smaller than the de Sitter horizon $H_{0}^{-1}$, then the wall worldsheet is well approximated by the future light cone of the center of spacetime symmetry of the bubble. We shall choose coordinates so that this center is at $\mathbf{x}=t=0$ and $\tau$ $=0$. Then, as $t \rightarrow \infty$, the bubble wall asymptotically approaches $|\mathbf{x}| \rightarrow H_{0}^{-1}$. The spacetime geometry of the bubble is illustrated in Fig. 5.

The relation between the coordinates $(t, \mathbf{x})$ and $(\tau, \xi)$ can be easily found if we assume, as is usually done, that (i) the potential $V(\phi)$ immediately outside the barrier has nearly the same value $V_{0}$ as in the false vacuum, and (ii) the gravitational effect of the bubble wall is negligible. Then, at sufficiently small values of $\tau$, the metric (14) inside the bubble is close to the open de Sitter metric, $a(\tau)=H_{0}^{-1} \sinh \left(H_{0} \tau\right)$. The coordinates $(t, \mathbf{x})$ and $(\tau, \xi)$ are related by the usual transformation between the flat and open de Sitter charts, which we shall not reproduce here.

We assume that markers are produced at a constant rate $r$ in the false vacuum outside the bubbles. The density of markers $n(\mathbf{x}, t)$ satisfies the equation

$$
\frac{\partial n}{\partial t}+3 H_{0} n=r
$$


which has a stationary solution $n_{0}=r / 3 H_{0}$. This de Sitterinvariant solution is rapidly approached, regardless of the initial conditions. We are interested in the average separation between the markers on the surfaces $\tau=\tau_{* i}$ corresponding to thermalization of the inflaton in the two types of bubbles. The densities of markers $n_{* i}$ on these surfaces are also constant, due to the spacetime symmetry of the bubbles (all points on the surface $\tau=$ const are equivalent).

The interior geometries of different types of bubble are nearly identical at early times (small $\tau$ ), while $V(\phi) \approx V_{0}$. Let us choose a value $\tau_{0}$ in that range and consider surfaces $\tau=\tau_{0}$. The geometry and the distribution of markers in the past of such surfaces are the same for the two types of bubble, and therefore the average separations of markers on these surfaces should also be the same, $d_{1}\left(\tau_{0}\right)=d_{2}\left(\tau_{0}\right)$ $\equiv d_{0}$. The separation of markers at thermalization is $d_{* i}$ $=Z_{* i} d_{0}$, where $Z_{* i}$ is the expansion factor between $\tau_{0}$ and $\tau_{* i}$, and we have

$$
N_{1} / N_{2}=\left(Z_{* 1} / Z_{* 2}\right)^{3} .
$$

To complete the calculation, we have to determine the fraction $p_{i}$ of the markers that end up in type- $i$ bubbles. Let $f_{i}(t)$ be the fraction of comoving volume occupied by type$i$ bubbles in a comoving volume which we assume to be free of bubbles at the initial moment $t=0$,

$$
f_{i}(0)=0 .
$$

The evolution of $f_{i}(t)$ is described by the equations

$$
\begin{aligned}
& \frac{\partial f_{1}}{\partial t}=\frac{4 \pi \lambda_{1}}{3 H^{3}}\left(1-f_{1}-f_{2}\right), \\
& \frac{\partial f_{2}}{\partial t}=\frac{4 \pi \lambda_{2}}{3 H^{3}}\left(1-f_{1}-f_{2}\right),
\end{aligned}
$$

where $\lambda_{i}$ is the nucleation rate of type- $i$ bubbles. In these equations we are neglecting "secondary" bubble nucleation which may occur within a comoving distance $H^{-1}$ from any given "primary" nucleation event, before the primary bubble reaches its asymptotic comoving size $H^{-1}$. Secondary nucleations will affect the comoving volume distribution very little, especially if the nucleation rate per unit volume is much smaller than $H^{4}$. This is a typical situation since the nucleation rates are usually exponentially suppressed. The solution of these equations with the initial condition (17) is

$$
f_{i}(t)=\frac{\lambda_{i}}{\lambda}\left[1-\exp \left(-\frac{4 \pi \lambda}{3 H^{3}} t\right)\right],
$$

where $\lambda=\lambda_{1}+\lambda_{2}$. The fraction of markers that end up in bubbles of type $i$ is given by

$$
p_{i}=f_{i}(t \rightarrow \infty)=\lambda_{i} / \lambda .
$$

Now, substituting Eqs. (16) and (21) into Eq. (8) for the probabilities we obtain

$$
\frac{P_{1}}{P_{2}}=\frac{\lambda_{1}}{\lambda_{2}}\left(\frac{Z_{* 1}}{Z_{* 2}}\right)^{3}
$$

This agrees with one's intuitive expectation that the probability should be proportional to the nucleation rate and to the volume expansion factor inside the bubbles.

\section{DISCUSSION}

In this paper we have suggested a cutoff procedure which allows one to assign probabilities to different types of thermalized region in an eternally inflating universe. The probabilities are calculated with the aid of "markers"imaginary pointlike objects which are assumed to be created at a constant rate in the inflating regions where the inflaton field is at the top of its potential. The probability for regions of type $i$ is then

$$
P_{i} \propto p_{i} N_{i},
$$

where $p_{i}$ is the fraction of markers that end up in type- $i$ regions and $N_{i}$ is the number of galaxies formed in a comoving sphere of radius equal to the average separation between the markers.

In contrast to some earlier proposals, the new prescription is manifestly gauge-invariant. It also gives the expected results in cases where we have well-motivated intuitive expectations for the probabilities.

The method of calculating probabilities presented in this paper has some similarities to the so-called $\epsilon$-prescription that was proposed in Ref. [19]. Starting with a comoving volume with $\phi$ near the top of the potential, the numbers of galaxies are calculated in this prescription by imposing cutoffs at different times in different types of region. The cutoff in type- $i$ regions is chosen at the time $t_{c i}$ when all but a small fraction $\epsilon$ of the comoving volume destined to thermalize in this type of region has thermalized. The value of $\epsilon$ is the same for all types of region, but the cutoff times are different. The limit $\epsilon \rightarrow 0$ is taken after calculating the probabilities. The $\epsilon$-prescription was applied in [20] to calculate the probabilities in open inflation and gave the same result (22) that we obtained here.

To see the connection between this prescription and the method of the present paper, imagine that the initial comoving region contains a large number of markers. If $p_{i}$ is the fraction of markers that are going to end up in type- $i$ regions, then the cutoffs in $\epsilon$-prescription are imposed at the times when the numbers of markers $\mathcal{N}_{m i}$ in the two types of region are related by $\mathcal{N}_{m 1} / \mathcal{N}_{m 2}=p_{1} / p_{2}$. The number of galaxies in each type of region is $\mathcal{N}_{g i}=\mathcal{N}_{m i} N_{i}$, where $N_{i}$ is the number of galaxies per one marker. Hence,

$$
\frac{P_{1}}{P_{2}}=\frac{\mathcal{N}_{g 1}}{\mathcal{N}_{g 2}}=\frac{p_{1}}{p_{2}} \frac{N_{1}}{N_{2}},
$$

which has the same form as Eq. (8).

One difference between the two methods is that markers are continuously produced in our new approach, while in $\epsilon$-prescription new markers are not produced even in regions 
when the inflaton field fluctuates back to the top of its potential. Another difference is that $\epsilon$-prescription uses constant- $t$ cutoffs, while the new approach uses spherical cutoffs. Because of its reliance on a constant $t$ cutoff, the results obtained using $\epsilon$-prescription are generally gaugedependent, whereas the new method is gauge independent.

The most straightforward way to implement the new prescription is through a numerical simulation of an eternally inflating spacetime. This method, however, suffers from severe computational limitations [11]. Alternatively, one can use an approximate analytic method based on the FokkerPlanck (FP) formalism of stochastic inflation $[7,21,4]$. This method works very well for the calculation of $p_{i}$ [22]. However, the results for the density of markers obtained using this approach are generally not gauge invariant.

The FP formalism can be used to calculate the physical volume $V_{* i}(t)$ that thermalizes prior to some time $t$ and the number of markers $N_{* i}(t)$ contained in that volume for different types of regions. The average distance between the markers can be expressed as

$$
d_{i}(t)=\left[\frac{V_{* i}(t)}{N_{* i}(t)}\right]^{1 / 3} .
$$

One might expect that in the limit $t \rightarrow \infty, d_{i}(t)$ should approach the average separation between markers on the thermalization surfaces $\Sigma_{* i}$ (calculated in large spherical volumes). However, this is not generally the case. The thermalized volume $V_{* i}(t)$ in an eternally inflating universe is dominated by the newly thermalized regions (shortly before the time $t$ ), and the density of markers in these regions is generally correlated with the choice of the time variable $t$.

The density of markers on the surfaces $\Sigma_{* i}$ can vary greatly from one area to another. In some places the field $\phi$ takes a more or less direct route from the top of the potential to $\phi_{* i}$, resulting in a relatively high density of markers, while in others it takes a long time fluctuating up and down the potential, so that the markers are greatly diluted. By including only regions that thermalize prior to time $t$, we "reward" regions that thermalize faster and therefore introduce a bias favoring higher densities of markers. This qualitative tendency is present for most choices of the time variable, but quantitatively the bias will not be the same. Hence, one should not be surprised that $d_{i}$ calculated from Eq. (25) depends on the choice of gauge.

Despite this gauge dependence, the FP method may give approximately valid results for some class of model. It has been argued in Refs. $[19,23]$ that in the case of $\epsilon$-prescription the gauge dependence is rather weak for a wide class of potentials. One can expect the situation to be similar for our new method when a constant $t$ cutoff is used. However, more work is needed to determine what additional requirements the potential should satisfy for this approximate gauge independence to apply.

We started in Sec. II of this paper by formulating a set of requirements that we believe any method for calculating the probabilities should satisfy. The specific prescription we introduced in Sec. III can be regarded as an "existence proof," demonstrating that a prescription satisfying all the criteria can indeed be constructed. It is quite possible, however, that our prescription is not unique and that more attractive and better motivated methods can be developed. With this in mind, we conclude by indicating some possible shortcomings and limitations of our method.

Admittedly, our prescription includes an element of arbitrariness when we assume that markers are produced only in regions where the inflaton $\phi$ is at the top of its potential. This " $\delta$-function" source should not be understood literally. The semiclassical picture of eternal inflation involves smearing over spacetime scales $\sim H^{-1}$ and over scalar field intervals $\sim H / 2 \pi$. Hence, marker formation at the top of the potential is equivalent to marker formation within an interval $\Delta \phi$ $\sim H / 2 \pi$ from the top of the potential. However, an attempt to extend marker formation to a wider interval encounters some ambiguities. Formally, there is no problem in calculating the density $n_{i}$ of markers in thermalized regions even if these are produced at some given rate $R(\phi) d \phi$ per unit proper time and volume in regions where the field is in the range $d \phi$. However, there is an obvious arbitrariness in what should be chosen as our "smearing function"' $R(\phi)$. Also, if markers are produced at different rates at different values of $H(\phi)$, the calculation of the fraction of markers $p_{i}$ that end up in region $i$ becomes somewhat ill posed. Markers formed at different values of $\phi$ will have a different probability $p_{i}$ of ending up in a type- $i$ region and it is not clear how to weigh the different contributions. One could calculate $p_{i}(\phi)$ and then average over $\phi$ with some weight, but it is not clear how the weight function is to be determined. It thus appears that confining marker formation to the top of the potential has some advantages and may be not as arbitrary as it first seems.

It is not clear whether or not our method will give reasonable results when applied to the most general type of inflationary scenario when the inflaton potential has several local maxima. Additional complications arise in models where the minimum of the potential has a non-vanishing cosmological constant. In such models, regions of true vacuum in the postinflationary universe may fluctuate back to the quantum diffusion range of the inflaton potential and the spacetime structure is more complicated than the one represented in Fig. 2.

Also, our method is not applicable when the potential is unbounded from above, in which case eternal inflation runs into the Planck boundary. This is not particularly worrisome, since after all the inflaton may be a modulus with a compact range, and the inflaton potential may well be bounded from above at a scale much lower than the Planck scale.

\section{ACKNOWLEDGMENTS}

It is a pleasure to thank Ken Olum, Masafumi Seriu, and especially Serge Winitzki for useful discussions. This work was supported by the Templeton Foundation under grant COS 253. J.G. is partially supported by CICYT, under grant AEN99-0766. A.V. is partially supported by the National Science Foundation. 
[1] For a review of inflation, see, e.g., A.D. Linde, Particle Physics and Inflationary Cosmology (Harwood Academic, Chur, Switzerland, 1990); K. A. Olive, Phys. Rep. 190, 307 (1990).

[2] A. Vilenkin, Phys. Rev. Lett. 74, 846 (1995).

[3] Related ideas have been discussed by B. Carter (unpublished); J. Leslie, Mind 101.403, 521 (1992); J. R. Gott, Nature (London) 363, 315 (1993); A. Albrect, in Birth of the Universe and Fundamental Physics, Lecture Notes in Physics Vol. 455 (Springer-Verlag, Berlin, 1995); and A. D. Linde et al. [4,5].

[4] A. D. Linde, D. A. Linde, and A. Mezhlumian, Phys. Rev. D 49, 1783 (1994).

[5] J. Garcia-Bellido, A. D. Linde, and D. A. Linde, Phys. Rev. D 50, 730 (1994).

[6] J. Garcia-Bellido and A. D. Linde, Phys. Rev. D 51, 429 (1995).

[7] A. Vilenkin, Phys. Rev. D 27, 2848 (1983).

[8] A. D. Linde, Phys. Lett. B 175, 395 (1986).

[9] A. H. Guth, Phys. Rep. 333, 555 (2000).

[10] A. Vilenkin, Phys. Rev. Lett. 81, 5501 (1998).

[11] V. Vanchurin, A. Vilenkin, and S. Winitzki, Phys. Rev. D 61,
083507 (2000).

[12] J. Garriga and A. Vilenkin, Phys. Rev. D 57, 2230 (1998).

[13] A. D. Linde, Phys. Lett. B 327, 208 (1994).

[14] A. Vilenkin, Phys. Rev. Lett. 72, 3137 (1994).

[15] P. Ginsparg and M. Perry, Nucl. Phys. B222, 245 (1983); R. Bousso and S. W. Hawking, Phys. Rev. D 54, 6312 (1996).

[16] J. R. Gott, Nature (London) 295, 304 (1982); J. R. Gott and T. S. Stalter, Phys. Lett. 136B, 157 (1984).

[17] M. Bucher, A. S. Goldhaber, and N. Turok, Phys. Rev. D 52, 3314 (1995); M. Bucher and N. Turok, ibid. 52, 5538 (1995).

[18] M. Sasaki, T. Tanaka, K. Yamamoto, and J. Yokoyama, Phys. Lett. B 317, 510 (1993); K. Yamamoto, M. Sasaki, and T. Tanaka, Astrophys. J. 455, 412 (1995).

[19] A. Vilenkin, Phys. Rev. D 52, 3365 (1995).

[20] A. Vilenkin and S. Winitzki, Phys. Rev. D 55, 548 (1997).

[21] A. A. Starobinsky, in Field Theory Quantum Gravity and Strings, Lecture Notes in Physics Vol. 246 (Springer, Heidelberg, 1986).

[22] J. Garriga, A. Vilenkin, and S. Winitzki (in preparation).

[23] S. Winitzki and A. Vilenkin, Phys. Rev. D 53, 4298 (1996). 